\title{
ERRATUM
}

\section{Thinking of the children: assessing archaeological evidence for childhood- ERRATUM}

Rebecca Redfern

Published by Cambridge University Press, 26 October 2018.

In this review article, the publication information regarding the book by Robin Derricourt, Unearthing childhood. Young lives in prehistory, was incorrect. The book is solely published by Manchester University Press and the price is $£ 20$.

\section{Full citation}

REDfern, R. 2018. Thinking of the children: assessing archaeological evidence for childhood. Antiquity 92: 1392-93. https://doi.org/10.15184/aqy.2018.205 